\title{
IMPROVEMENT OF THE OPTICAL REFERENCE SYSTEM BY PHOTOGRAPHIC ASTROMETRY: \\ FIRST RESULTS OF SIMULATIONS WITH GLOBAL BLOCK ADJUSTMENT METHODS
}

\author{
N. ZACHARIAS \\ Hamburger Sternwarte, Gojenbergsweg 112 \\ 2050 Hamburg 80, FRG
}

\begin{abstract}
Block adjustment (BA) simulations of an entire hemisphere show major improvements in individual as well as systematic accuracy of star positions obtained by photographic astrometry, independent of systematic errors of the reference star catalog used. Results for the accuracy estimates obtained from a patch-like plate pattern in the sky are not valid for closed plate pattern. The importance of BA methods for the realisation of a reference system is stressed.
\end{abstract}

\section{Introduction}

A review of the principles and applications of the simultaneous adjustment of overlapping plates, known as block adjustment (BA), has been published recently (Eichhorn 1988). The algorithm adopted here is based on that of Googe et.al. (1970); further details can be found elsewhere (Zacharias 1987).

This paper deals only with simulations, but the data structure of the CPC2 project (Nicholson et.al. 1984, Zacharias et.al. 1991) is widely used. Contrary to most other investigations of the accuracy obtainable with a BA, we investigate here a global pattern, i. e. a closed zone and an entire hemisphere, following Ebner (1970). Furthermore, typical systematic errors of the current FK5 based system (Morrison et.al. 1990), depending on the coordinates in the sky, are introduced to show their influence on the positions obtained from a BA solution.

\section{Cape Zone Simulation}

For a first investigation, the data structure of the Cape Zone of the CPC2 was used, with details listed in Table 1. The positions of that catalog are defined as error free (true) and then projected onto the corresponding plates. Random errors with normal 
distribution $N(\sigma, 0)$ are added to the $x, y$-coordinates as well as to the reference star positions according to Table 1 .

Furthermore, a constant offset is added to the declinations of all reference stars within $-50^{\circ} \leq \delta \leq-48^{\circ}$, thus

$$
\delta_{\text {ref.cat }}:=\delta_{\text {true }}+N\left(\sigma_{\text {ref }}, 0\right)+100 \text { mas }
$$

to simulate a systematic error in the reference star catalog.

Results of a BA with appropriate weights but no additional constraints on the 8 parameter plate model (full linear + plate tilt terms) are summarized in Table 2. Fig. 1 shows the mean of the declination differences (BA-true), taken over all stars along right ascension. The amplitude of the systematic error is reduced by about a factor of 3. Similar calculations with the Cape Zone, adding long periodic systematic waves along right ascension, show that these could not be removed by BA methods. Details can be found elsewhere (Zacharias 1987).

\section{Global I}

This first test covering an entire hemisphere with a 4-fold overlap pattern uses a large plate size to minimize CPU time. In addition to the plate model already mentioned, an orthogonal plate model was used as well. Details are given in Table 1 and 2. The following systematic error in declination was added to the reference star positions for those stars within the RA range of $6^{h} \leq \alpha \leq 12^{h}$ :

$$
\Delta \delta_{\text {sys.err. }}=150 \text { mas } \cos (4 \delta)
$$

The resulting declination differences (ref.cat.-true) and (BA-true) for the 8 and 4 parameter plate model are displyed in Fig. 2,3 and 4 respectively. Note the amplitude of the systematic error is reduced by a factor of about 3 and 6 respectively in the two BA solutions!

\section{Global II}

The full CPC2 data structure with 5695 plates is used in the Global II simulation. Similar to Global I, a systematic error was added to the reference star positions, now for those stars within the RA range of $6^{h} \leq \alpha \leq 9^{h}$ :

$$
\Delta \delta_{\text {sys.err }}=150 \text { mas } \cos (8 \delta)
$$

For all reference stars within this $\mathrm{RA}$ range, the resulting differences (BA-true) and (ref.cat.-true) are combined in Fig.5. The corresponding differences (BA-true) for all stars of this RA range are shown in Fig. 6. The amplitude of the systematic error is reduced by at least a factor of 10 in the BA solution, despite the small plate size and large number of plates used here. Note also the very low value for $\sigma(B A-$ true $)$ which is even below $\sigma_{x y} / \sqrt{n}$ due to the powerful constraint set by the global closed pattern of plates (Ebner 1970). 


\section{Conclusions}

A BA of a global closed 4-fold overlap pattern of plates gives a further major improvement in random and systematic accuracy of star positions as compared to BA results obtained from smaller unclosed patches of overlapping plates. Catalog accuracies below $\sigma_{x y} / \sqrt{n}$ are possible even with a large number of plates (many thousands).

We like to stress the potential of current photographic astrometry for the homogenisation of the present reference system on scales of about $20^{\circ}$, independent of transit circle observations. A complete and homogeneous catalog for all stars down to about $13^{m}$ could be established by these means. More details can be found elsewhere (de Vegt 1988).

Furthermore the BA results seem to be largely independent of the plate size. Thus a future dedicated astrometric telescope (de Vegt 1989) with only a $2^{\circ}$ field but which will reach magnitude $18^{m}$, could provide a direct link to the extragalactic reference frame, independent of present reference star catalogs (Clube 1968) which will be used only for the conventional single plate adjustment to provide starting values for the final BA.

Of course all results presented here are valid only when there are no systematic errors in the photographic data. Using modern techniques and a calibration with quasi error-free positions from a future HIPPARCOS catalog, it will be possible to meet these requirements to at least one micron level of precision.

\section{Acknowledgements}

The author thanks Prof. Dr. K. Hasselmann, director of the DKRZ at Hamburg, for allocation of computer time on the $\mathrm{Cray}_{\text {-2S }}$. The friendly assistance of the advisory team at the DKRZ is gratfully acknowledged. Comments and corrections on the draft paper by A.Murray and L.Morrison have been very helpful.

\section{References}

Clube, S.V.M.: 1968, In: Highlights of Astronomy, Dordrecht (Reidel), pp. 347-351

de Vegt, Chr.: 1988, In: Mapping the Sky, IAU Symp. 133, 211

de Vegt, Chr.: 1989, In: Star catalogues: A centennial tribute to A.N. Vyssotsky, Contr. Van Vleck Obs. 8, 51

Ebner, H.: 1970, Astron. Nachr. 292, 65

Eichhorn, H.: 1988, In: Mapping the Sky, IAU Symp. 133, 177

Googe, W. D., Eichhorn, H., Lukac, C. F.: 1970, Monthly Notices Roy. Astron. Soc. 150, 35

Morrison,L.V., Argyle,R.W., Helmer,L., Fabricius,C.,

Einicke, O.H., Quijano,L., Muinos,J.L.: 1990, In:

Inertial coordinate systems on the sky, IAU Symp. 141, 391

Nicholson, W., Penston, M. J., Murray, C. A., de Vegt, Chr.: 1984, Monthly Notices Roy. Astron. Soc. 208, 911

Zacharias, N.: 1987, Ph.D. Thesis, University of Hamburg

Zacharias, N., de Vegt, Chr.: 1991, in prep. for Astron. Astrophys. 

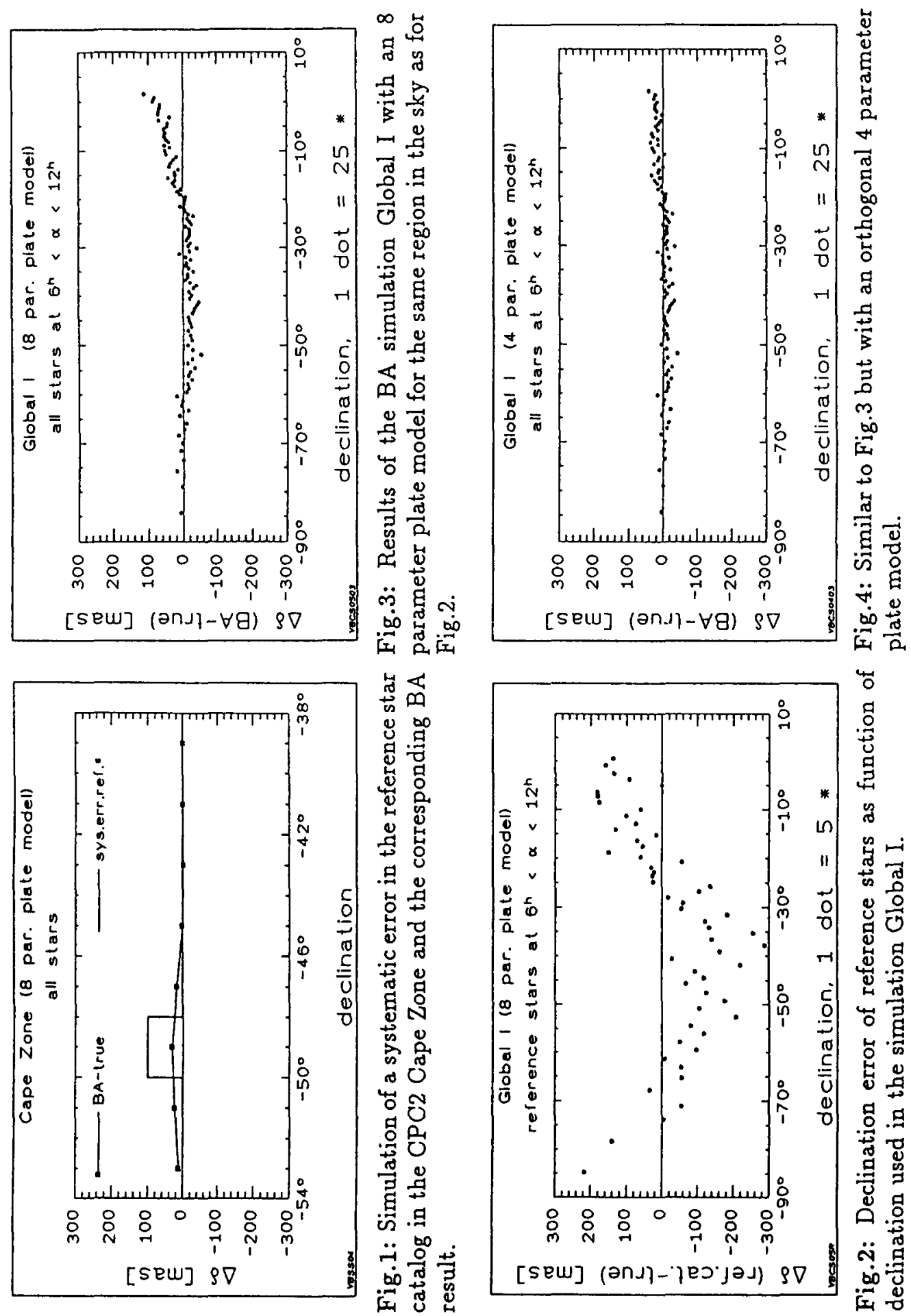

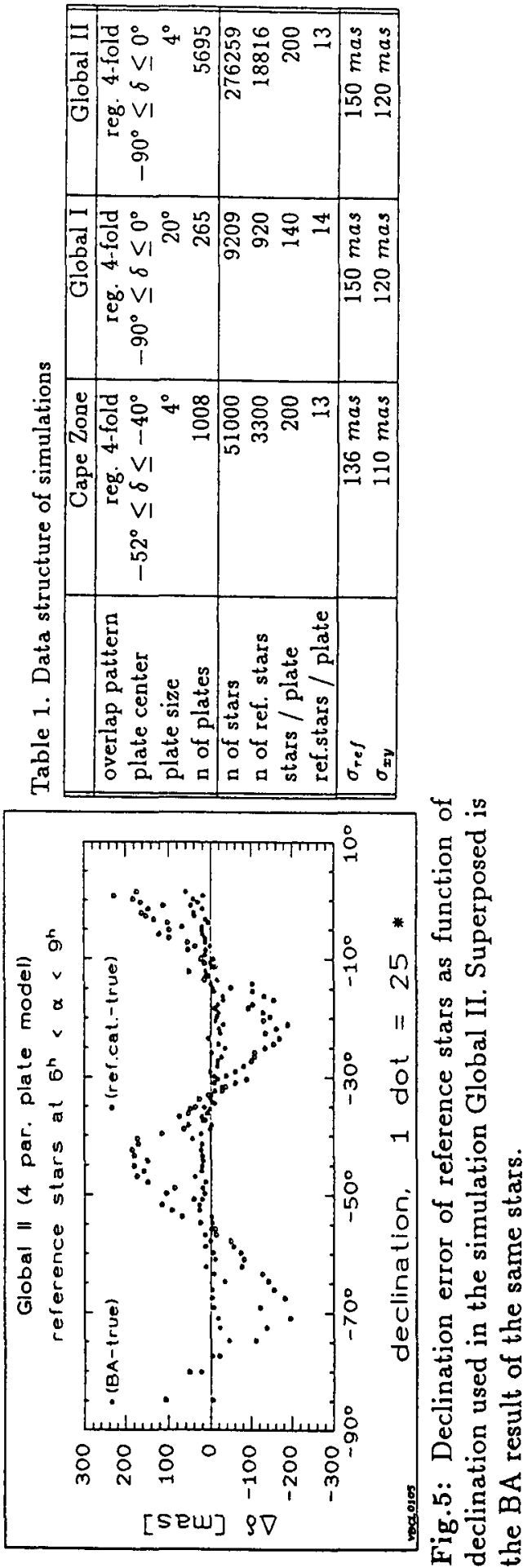
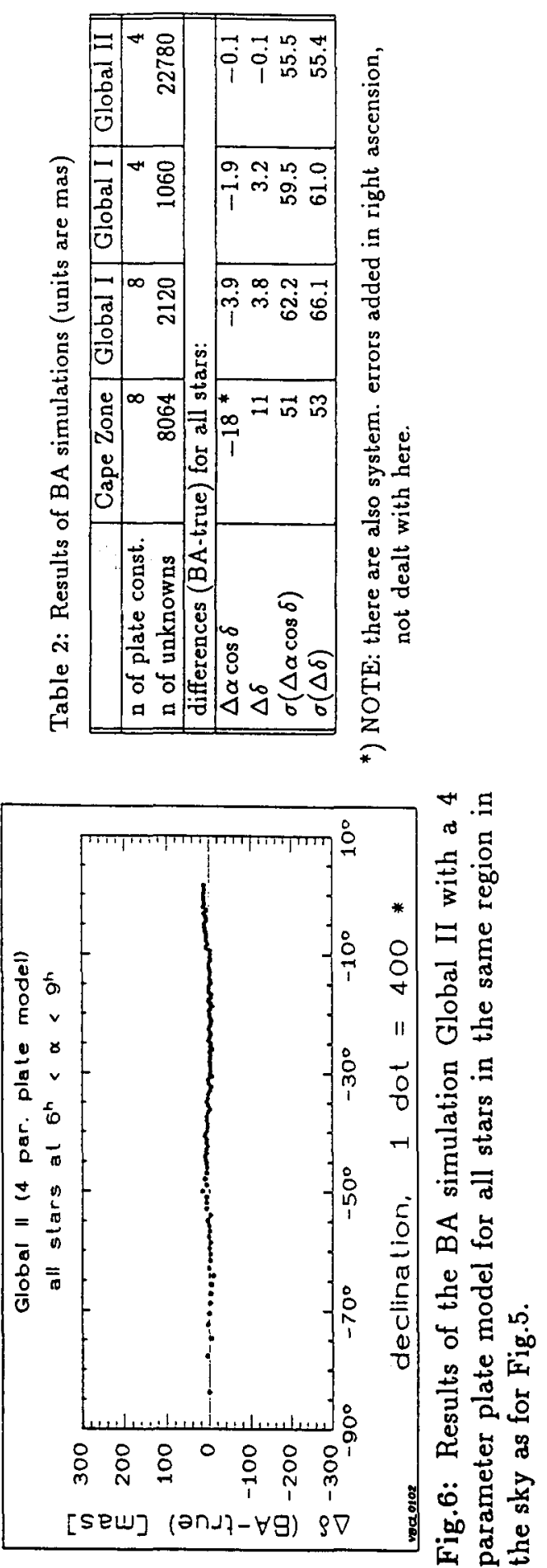